
\title{
25 Research Soure \\ Predictive Utility of Changes in Optic Nerve Sheath Diameter After Cardiac Arrest for Neurologic Outcomes
}

\section{Heekyung Lee}

Hanyang University Graduate School of Medicine: Hanyang University College of Medicine, Hanyang University Seoul Hospital

\section{Joonkee Lee}

Hanyang University Guri Hospital, Hanyang University Medical School: Hanyang University College of Medicine

\section{Hyungoo Shin ( $\nabla$ seodtst@gmail.com )}

Hanyang University College of Medicine https://orcid.org/0000-0003-3203-1672

\section{Original research}

Keywords: Heart arrest, optic nerve sheath diameter, Patient outcome assessment

Posted Date: March 2nd, 2021

DOI: https://doi.org/10.21203/rs.3.rs-265366/v1

License: (c) (i) This work is licensed under a Creative Commons Attribution 4.0 International License. Read Full License 


\section{Abstract}

Background: Optic nerve sheath diameter (ONSD) is effective in predicting the neurologic outcome of patients with post-cardiac arrest (CA) return of spontaneous circulation. This study aimed to investigate the effect of ONSD changes before and after CA on neurologic outcomes in patients with return of spontaneous circulation after CA using brain computed tomography (CT).

Methods: We included patients who were hospitalized after CA and underwent pre- and post-CA brain CT from January 2001 to September 2020. They were divided into the good and poor neurologic outcome (GNO and PNO, respectively) groups based on the neurologic outcome at hospital discharge. We performed between-group comparisons of the amount and rate of post-CA changes on brain CT. Moreover, we calculated the area under the curve to determine the predictive value of ONSD changes for neurologic outcomes.

Results: We included 96 enrolled patients; among them, 25 had GNO. The amount of change was significantly higher in the PNO group than in the GNO group ( 0.63 vs. $0.30 \mathrm{~mm} ; p=0.030)$. Moreover, there was a higher rate of change in the PNO group than in the GNO group (12.29 vs. $5.26 \% ; p=0.041)$. The AUC for predicting PNO was $0.64(95 \% \mathrm{Cl}=0.53-0.73 ; p=0.04)$ and patients with a rate of ONSD change $>27.2 \%$ had PNO with specificity and positive predictive values of $100 \%$.

Conclusion: The amount and rate of ONSD changes on brain CT were significantly associated with the neurologic outcomes in post-CA patients. ONSD changes may predict neurologic outcomes in post-CA patients.

\section{Introduction}

Ischemia/reperfusion cerebral injury after cardiac arrest (CA) may cause cerebral edema.[1, 2] This results in an increase in intracranial pressure (ICP) and contributes to poor neurologic outcomes in patients with post-CA return of spontaneous circulation (ROSC). $[3,4]$ In these survivors, there is a need for early detection of increasing ICP and neurologic outcome prediction to allow appropriate post-resuscitation care.[5] This allows priority to be given to patients with expected good neurologic outcomes given the limited medical resources. There have been studies on various predictive factors for post-CA neurologic outcomes, including neurologic examination of brainstem reflexes, electrophysiological tests, and serum biomarkers, such as neuron specific enolase and S-100B.[6-8] However, these factors have been recommended as prognostic factors at 72 post-CA hours.[8-10] Moreover, early brain computed tomography (CT) of patients with post-CA ROSC may play a crucial role as a prognostic predictor. Further, the American Heart Association guidelines recommend early post-CA brain CT scans and confirm that a decrease in the grey-to white matter ratio (GWR) can help predict neurologic outcome.[10-13] Additionally, there have been studies with optic nerve sheath diameter (ONSD) on brain CT for predicting neurologic outcomes in post-CA survivors.[14-16] 
Previous studies have shown that the ONSD on brain CT could be a useful tool as a non-invasive method of ICP measurement.[17, 18] Additionally, recent studies have demonstrated that the ONSD on brain CT is useful for early neurologic outcome prediction through evaluation of increased ICP in patients with postCA ROSC. $[15,19]$ A recent meta-analysis confirmed the utility of ONSD as a prognostic factor for neurologic outcomes in post-CA patients.[20] However, most of the studies included in the meta-analysis indicated that sole use of ONSD had limited predictive utility for prognosis. Furthermore, all these studies only measured the post-CA ONSD without considering individual differences.

This study aimed to assess differences between pre- and post-CA ONSD in patients with ROSC after CA using brain CT imaging. Additionally, we aimed to investigate the impact of the amount and rate of postCA ONSD changes on the neurologic outcome at discharge.

\section{Methods}

\section{Study design and population}

This retrospective observational cohort study investigated brain CT scans of patients hospitalized after CA who visited the emergency department of a single university-affiliated hospital in Korea between January 2001 and September 2020. This study was approved by the Institutional Review Board of Hanyang University Guri Hospital (IRB No. GURI 2020-12-008), which waived the requirement of informed consent.

We included adult patients hospitalized after CA who underwent pre- and post-CA brain CT. The exclusion criteria were as follows: (1) being transferred to another hospital after ROSC, (2) age< 19 years, (3) having traumatic/non-traumatic brain hemorrhage or brain tumor, (4) a history of ophthalmological disorders or surgeries that could affect ONSD, and (5) having the most recent pre-CA brain CT obtained at an age $<19$ years. Finally, eligible patients were divided into the GNO (good neurologic outcome) and PNO (poor neurologic outcome) groups based on the neurologic outcome at discharge; subsequently, we measured ONSD changes and performed between-group analysis. The primary outcome was the association between ONSD changes and the neurologic outcomes of patients hospitalized after CA.

\section{Data collection}

We retrospectively collected the following data from electronic medical records: age, gender, comorbidities (hypertension, diabetes, myocardial infarction), etiology (cardiac, respiratory), location of $\mathrm{CA}$, whether the CA was witnessed, bystander CPR, first monitored shockable rhythm, CA duration including the no-flow (time between CA and CPR initiation) and low-flow time (time between active CPR and ROSC), and administered targeted temperature management (TTM). Based on the medical records, we determined the interval between the latest pre-CA brain CT and ROSC (month), which was termed as 'CT to ROSC', and between ROSC and post-CA brain CT (minute), which was termed as 'ROSC to CT'. Additionally, we collected data regarding the neurologic outcomes on discharge using the Glasgow- 
Pittsburgh Cerebral Performance Categories (CPC). Based on the CPC scale, we defined good (GNO) and poor neurologic outcomes (PNO) as a CPC 1 or 2 and 3-5, respectively.

\section{ONSD measurements using brain CT}

Brain CT scans were performed based on standard protocols using non-contrast 4-mm contiguous slices parallel to the orbital floor from the skull base to the vertex. The pre-CA and post-CA ONSDs were bilaterally measured at $3 \mathrm{~mm}$ behind the globe on brain CT using the picture archiving and communication system (PACS) ruler tool (PiView STAR, INFINITT, Seoul, Korea). Images were magnified at $450 \%$ and changed to the 'mediastinum' window (window width: 440; window level: 45) using a PACS tool. The ONSDs of the right and left eyes were averaged to obtain the mean value. All measurements were performed using emergency physicians blinded to the patient information, including neurologic outcome. Additionally, we calculated the amount and rate of ONSD change. We defined the amount of change as the difference between the pre-CA and post-CA ONSD. Moreover, the rate of ONSD change was calculated as follows:

\section{$\left(\frac{\text { Post-CA ONSD - Pre-CA ONSD }}{\text { Pre-CA ONSD }}\right) \times 100$}

We used the following three CT equipment: Somatom Sensation 16, SOMATOM Definition DS, and SOMATOM Definition Edge (Siemens Healthcare, Erlangen, Germany). The following parameters were used: 120 kVp, 250-500 mAs, and 4 to 4.5-mm slice thickness. All CT images were stored as the Digital Imaging and Communication in Medicine format in the PACS.

\section{Sample size}

We calculated the sample size based on a pilot study on 33 participants using G*Power (3.1.9.6; Heine Heinrich University, Düsseldorf, German). The mean ONSD of patients with GNO and PNO was $4.75 \pm 1.45$ $\mathrm{mm}$ and $5.63 \pm 1.85 \mathrm{~mm}$, respectively. The required sample size was calculated as 90 participants (effect size: 0.53, a-error: 0.05, power: 0.8); finally, considering a 10\% drop-out rate, 99 participants were required.

\section{Statistical analysis}

Continuous and categorical variables were reported as the median with interquartile range (IQR) and number with percentages. Normally distributed variables were analyzed using the Mann-Whitney U-test and Wilcoxon rank-sum test while non-normally distributed variables were analyzed using the ShapiroWilk test. Chi-square tests or Fisher's exact test were used to analyze categorical variables. Statistical significance was set at $P<0.05$. Multivariable analysis with logistic regression was used to determine the risk factors for poor neurologic outcomes with adjustment for confounding variables found significant on univariate analysis. Variables with $p<0.2$ on univariate analysis with the rate of ONSD change were included in the multivariable analysis. Further, the Hosmer-Lemeshow test was used to confirm the logistic model calibrations. The predictive performance of the main outcome was assessed using the area under the receiver operating characteristic ([ROC] AUC) of the sensitivity over 1 - specificity. Results were obtained using the Youden index and presented as a 95\% confidence interval (CI) of AUC with 
sensitivity, specificity, positive predictive value (PPV), and negative predictive value (NPV). ROC analysis was performed using MedCalc Statistical Software (version 17.2, MedCalc Software, Ostend, Belgium) while the other statistical analyses were performed using SPSS software (version 25.0, IBM, Armonk, NY).

\section{Results}

\section{Baseline characteristics}

Among 145 post-CA survivors who underwent brain CT before and after CA, 49 patients were excluded as follows: 40 patients who transferred to another hospital, seven patients with intracranial or subarachnoid hemorrhage, one patient with a brain tumor, and one patient aged $\leq 18$ years. Finally, we enrolled 96 patients and allocated them to the GNO group $(n=25,26.0 \%)$ and PNO group $(n=71,74.0 \%)$ (Fig. 1).

Table 1 summarizes the demographic and clinical characteristics. The median age of the included patients was 70 (IQR: $58-79$ ) years with $56.3 \%$ being male. The GNO group was significantly younger than the PNO group. Moreover, the GNO group showed a significantly higher frequency of cardiac etiology and shockable rhythm, as well as a shorter no-flow and low-flow time, than the PNO group. Contrastingly, out-of-hospital cardiac arrest was more frequent in the PNO group. 
Table 1

Baseline characteristics of enrolled patients.

\section{Total $(n=96) \quad$ GNO $(n=25) \quad$ PNO $(n=71) \quad p$-value}

Demographics

Age, year

Gender, male

$70(58-79)$

$60(52-67)$

$75(61-80)$

$<0.001$

$54(56.3)$

$15(60.0)$

$39(54.9)$

0.660

Comorbidities

HTN

DM

MI

Etiology

Cardiac

Respiratory

Others

Resuscitation

Location of arrest, OHCA

Witnessed

Bystander CPR

Shockable rhythm

No-flow time, $\mathrm{min}$

Low-flow time, min

TTM

CT to ROSC interval ${ }^{\star}$, month

ROSC to CT interval ${ }^{\dagger}$, min
52 (54.2)

37 (38.5)

$16(16.7)$
$14(56.0)$

$6(24.0)$

4 (16.0)

$14(56.0)$

8 (32.0)

$3(12.0)$

$33(34.4)$

76 (79.2)

72 (75.0)

61 (63.5)

11 (11.5)

$10(0-21)$

$10(6-16)$

$6(6.3)$

$27(6-55)$

$104(51-171)$
$16(64.0)$

19 (76.0)

$18(72.0)$

8 (32.0)

$4(0-9)$

$6(3-10)$

3 (12.0)

$40(6-55)$

$60(33-118)$
38 (53.5)

31 (43.7)

12 (16.9)

9 (12.7)

32 (45.1)

30 (42.3)

0.006

Abbreviations: $\mathrm{GNO}=$ good neurologic outcome; $\mathrm{PNO}=$ poor neurologic outcome; $\mathrm{HTN}$ = hypertension; $\mathrm{DM}=$ diabetes mellitus; $\mathrm{MI}=$ myocardial infarction; $\mathrm{OHCA}=$ out-of-hospital cardiac arrest; $\mathrm{CPR}=$ cardiopulmonary resuscitation; TTM = targeted temperature management; $\mathrm{CT}$ = computed Tomography; ROSC = return of spontaneous circulation.

* The interval between the latest pre-CA brain CT and ROSC.

† The interval between ROSC and post-CA brain CT. 


\section{Comparison of pre-CA and post-CA ONSDs}

In both groups, the post-CA ONSD was significantly higher than the pre-CA ONSD (Fig. 2). In the GNO group, there was a significant difference between the pre-CA ONSD and post-CA ONSD (5.06 vs. $5.50 \mathrm{~mm}$, $p<0.001)$ in the GNO group. Similarly, in the PNO group, there was a significant difference between the pre-CA ONSD and post-CA ONSD (5.07 vs. $5.72 \mathrm{~mm}, p=0.001$ ) (Supplemental Table 1).

\section{The association between ONSD changes and neurologic outcomes}

Table 2 presents the between-group comparisons of the amount and rate of ONSD changes. There was no significant between-group difference in pre-CA ONSD ( $5.06 \mathrm{vs.} 5.07 \mathrm{~mm}, p=0.967)$ and post-CA ONSD (5.50 vs $5.72 \mathrm{~mm}, p=0.075$ ). However, the amount of ONSD change in the GNO group was significantly lower than that in the PNO group ( 0.30 vs. $0.63 \mathrm{~mm}, p=0.030)$. Additionally, the rate of ONSD change in the GNO group was significantly lower than that in the PNO group (5.26 vs. $12.29 \%, p=0.041$ ).

Multivariable analysis revealed no independent association between the rate of ONSD change and poor neurologic outcome $(\mathrm{OR}=1.075 ; 95 \% \mathrm{Cl}=0.990-1.167, p=0.084)$ (Table 3$)$.

Table 2

The comparisons of the amount and rate of ONSD changes between good and poor neurologic outcome.

\begin{tabular}{|c|c|c|c|c|}
\hline & Total $(n=96)$ & GNO $(n=25)$ & PNO $(n=71)$ & p-value \\
\hline \multicolumn{5}{|c|}{ Optic nerve sheath diameter } \\
\hline Pre-CA, mm & $5.07(4.73-5.52)$ & $5.06(4.76-5.53)$ & $5.07(4.73-5.52)$ & 0.967 \\
\hline Post-CA, mm & $5.66(5.41-6.01)$ & $5.50(5.16-5.88)$ & $5.72(5.49-6.04)$ & 0.075 \\
\hline \multicolumn{5}{|c|}{ Optic nerve sheath diameter changes between pre-CA and post-CA } \\
\hline Amount of change, $\mathrm{mm}$ & $0.57(0.25-0.84)$ & $0.30(0.18-0.65)$ & $0.63(0.32-0.87)$ & 0.030 \\
\hline Rate of change, $\%$ & $11.10(4.70-17.21)$ & $5.26(3.85-14.15)$ & $12.29(5.83-18.74)$ & 0.041 \\
\hline
\end{tabular}


Table 3

Multivariable logistic regression analysis for poor neurologic outcome with baseline variables and rate of optic nerve sheath diameter change.

\begin{tabular}{|llc|}
\hline Variables & Adjusted OR $(95 \%$ Cl $)$ & p-value \\
\hline Age, year & $1.115(1.031-1.206)$ & 0.006 \\
\hline DM & $3.358(0.636-17.733)$ & 0.154 \\
\hline Shockable rhythm & $0.084(0.008-0.911)$ & 0.042 \\
\hline No-flow time, min & $1.113(1.003-1.235)$ & 0.043 \\
\hline Low-flow time, min & $1.123(1.024-1.231)$ & 0.013 \\
\hline TTM & $0.119(0.008-1.794)$ & 0.124 \\
\hline Location of arrest, OHCA & $0.833(0.115-6.014)$ & 0.856 \\
\hline ROSC to CT interval ${ }^{*}$ min & $0.999(0.999-1.000)$ & 0.086 \\
\hline $\begin{array}{l}\text { Etiology, cardiac } \\
\text { Rate of change, \% }\end{array}$ & $0.080(0.012-0.558)$ & 0.011 \\
\hline $\begin{array}{l}\text { Abbreviations: OR = odds ratio; DM = diabetes mellitus; TTM = targeted temperature management; } \\
\text { OHCA = out-of-hospital cardiac arrest; ROSC = return of spontaneous circulation; CT = computed } \\
\text { tomography. }\end{array}$ & $1.075(0.990-1.167)$ \\
* The interval between ROSC and post-CA brain CT. & 0.084 \\
\hline
\end{tabular}

\section{Diagnostic value of ONSD changes for predicting the neurologic outcome}

The AUC for predicting PNO was $0.64(95 \% \mathrm{Cl}=0.53-0.73 ; p=0.04)$ in the ROC curve for the rate of ONSD change (Fig. 3). Patients with a rate of ONSD change $>27.2 \%$ had PNO with specificity and PPV of $100 \%$. GNO could be predicted using a cut-off value of $\leq 5.83 \%$ in the ROC curve for the rate of ONSD change, with a sensitivity and specificity of $60.0 \%$ and $76.06 \%$, respectively. The PPV and NPV were $46.9 \%$ and $84.4 \%$, respectively (Table 4 ). 
Table 4

Cut-off and diagnostic value of optic nerve sheath diameter change for predicting good and poor neurologic outcome.

\begin{tabular}{|llllll|}
\hline & Cut-off, \% & Sensitivity & Specificity & PPV & NPV \\
\hline Rate of change for predicting PNO & $>27.2$ & 0.085 & 1.000 & 1.000 & 0.278 \\
\hline Rate of change for predicting GNO & $\leq 5.83$ & 0.600 & 0.761 & 0.469 & 0.844 \\
\hline $\begin{array}{l}\text { Abbreviations: PPV = positive predictive value; NPV } \\
\text { outcome; GNO = good neurologic outcome. }\end{array}$ & \multicolumn{5}{l}{ negative predictive value; PNO = poor neurologic } \\
\hline
\end{tabular}

\section{Discussion}

This study found that the amount and rate of ONSD change were significantly associated with neurologic outcomes. Further, there was no significant between-group difference in the post-CA ONSD. However, there was no independent association of the rate of ONSD change with neurologic outcomes after adjusting for confounding variables. Together with other established predictors, the rate of ONSD change may be useful for predicting neurologic outcomes. To our knowledge, this is the first study to investigate individual differences in ONSD changes among post-CA survivors.

Previous studies have reported an association of neurologic outcomes in critically ill patients, including post-CA survivors, with increased ICP.[3, 4, 21, 22] The optic nerve sheath covers the optic nerve and is comprised of a subarachnoid space layer filled with cerebrospinal fluid (CSF).[23] ICP is positively correlated with the CSF pressure and ONSD length.[23, 24] ONSD is a potential non-invasive ICP estimator and could be useful for assessing intracranial hypertension.[24] In patients with post-CA hypoxic cerebral injury, increased ICP is associated with neurologic outcome.[3, 21, 22]

Several studies have reported that ONSD can predict neurologic outcomes in post-CA survivors. A retrospective cohort study from Korea reported an association of longer ONSD on initial brain CT with poor neurologic outcome.[15] Chelly et al. demonstrated that ONSD could be an early prediction tool for outcomes in post-CA patients treated using TTM.[19] Other studies have used ONSD combined with other predictors, including GWR or albumin levels, to enhance the predictive value. Moreover, a recent metaanalysis reported that ONSD could be useful for predicting neurologic outcome.[14, 20, 21] Inconsistent with these findings, a registry-based multicenter study reported no correlation between ONSD on early unenhanced brain CT and neurologic outcome in post-CA survivors managed using TTM.[25] Previous studies have reported that predicting neurologic outcomes in post-CA survivors using post-CA ONSD alone is insufficient with limitations.

ONSD can allow non-invasive ICP measurement and could serve as a surrogate marker for increased ICP. $[17,18]$ However, in healthy adults, there are differences in the baseline ONSD according to individual characteristics, including sex, body mass index (BMI), race, or eyeball size.[26, 27] Most studies of healthy volunteers have reported that the mean ONSD ranges from about $3 \mathrm{~mm}$ to $5 \mathrm{~mm}$; moreover, the reported 
mean or median ONSD values have varied across study cohorts depending on the race or measurement tools.[26-29] Ultrasonographic evaluation of healthy Asians revealed a longer ONSD in males and individuals with high BMI.[26, 27] Therefore, these individual differences could confound the interpretation of post-CA ONSD; moreover, it may be useful to consider the baseline ONSD for improving the prognostic value. Therefore, ONSD changes may be useful markers for ICP measurement changes. A prospective observational study on ONSD changes in patients with hydrocephalus reported a significant reduction in ONSD after ventriculoperitoneal shunt operation.[30] In our study, ONSD changes were more reliable than the ONSD itself for predicting neurologic outcomes in patients with post-CA.

A recent meta-analysis reported that compared with $\mathrm{CT}$ and $\mathrm{MR}$, sonographic measurement allowed more accurate prediction of neurologic outcome in patients with post-CA.[20] However, obtaining and comparing pre-CA and post-CA ONSD using ultrasound could be limited in clinical settings. Moreover, determining the pre-CA ONSD using brain MR has limitations given its specific modality. Recent studies have indicated that the axial proton density/T2-weighted turbo spin-echo fat-suppressed sequence is required for ONSD measurements using MR. However, in most post-CA patients, the T2-TSE image is not included in the diffusion-weighted MR.[31, 32] Additionally, there is a strong association of ONSD with eyeball transverse diameter (ETD) and ONSD/ETD ratio in healthy adults.[33] There is a need for further studies on the association between ONSD/ETD ratio and neurologic outcome in post-CA patients.

This study has several limitations. First, this was a single-center study with a limited sample size that led to an insufficient statistical power; however, we calculated the sample size, which was relatively large compared with that of other studies. Second, this retrospective study included patients who underwent both pre- and post-CA brain CT, which could lead to selection bias affecting the results. Third, although we attempted to extensively collect variables, there could be hidden confounders. Fourth, there could have been minor measurement errors given the very small size of the ONSD in brain CT. However, to minimize these errors, two blinded emergency physicians performed measurements using a standardized method with consensus. Fifth, current guidelines recommend neurologic outcome assessment at 3 months after discharge. However, we measured the neurologic outcome at discharge and did not determine the longterm outcome. Sixth, this was a retrospective study and the clinical utility of the predictive value for prognosis remains unclear. There is a need for large-scale prospective studies to enhance our findings.

\section{Conclusions}

The rate and amount of ONSD changes on brain CT were significantly associated with neurologic outcome in patients with post-CA. ONSD changes may be useful to predict neurologic outcome in patients with post-CA.

\section{Abbreviations}

ONSD, optic nerve sheath diameter; CA, cardiac arrest; CT, computed tomography; GNO, good neurologic outcome; PNO, poor neurologic outcome; ICP, intracranial pressure; ROSC, return of spontaneous 
circulation; GWR, grey-to white matter ratio; CPR, cardiopulmonary resuscitation; TTM, targeted temperature management; CPC, Cerebral Performance Categories; PACS, picture archiving and communication system; IQR, interquartile range; $A U C$, area under curve; ROC, the receiver operating characteristic; $\mathrm{Cl}$, confidence interval; PPV, positive predictive value; NPV, negative predictive value; CSF, cerebrospinal fluid; BMI, body mass index; ETD, eyeball transverse diameter

\section{Declarations}

\section{Acknowledgments}

This work was supported by the research fund of Hanyang University (HY-2021).

\section{Authors' contributions}

Heekyung Lee: Software, Formal analysis, Investigation, Resources, Data Curation, Writing - Original Draft. Joonkee Lee: Investigation, Resources, Data Curation, Writing - Original Draft. Hyungoo Shin: Conceptualization, Methodology, Software, Validation, Writing - Review \& Editing, Supervision.

\section{Funding}

None

\section{Availability of data and materials}

The datasets used and analyzed during the current study are available from the corresponding author on reasonable request.

\section{Ethics approval and consent to participate}

This study was approved by the Institutional Review Board of Hanyang University Guri Hospital (IRB No. GURI 2020-12-008), which waived the requirement of informed consent.

\section{Consent for publication}

Not applicable

\section{Competing interests}

The authors declare no competing interests.

\section{References}

1. Gueugniaud PY, Garcia-Darennes F, Gaussorgues P, Bancalari G, Petit P, Robert D. Prognostic significance of early intracranial and cerebral perfusion pressures in post-cardiac arrest anoxic coma. Intensive Care Med 1991;17:392-8. 
2. Sundgreen C, Larsen FS, Herzog TM, Knudsen GM, Boesgaard S, Aldershvile J. Autoregulation of cerebral blood flow in patients resuscitated from cardiac arrest. Stroke 2001;32:128-32.

3. Metter RB, Rittenberger JC, Guyette FX, Callaway CW. Association between a quantitative CT scan measure of brain edema and outcome after cardiac arrest. Resuscitation 2011;82:1180-5.

4. lida K, Satoh H, Arita K, Nakahara T, Kurisu K, Ohtani M. Delayed hyperemia causing intracranial hypertension after cardiopulmonary resuscitation. Crit Care Med 1997;25:971-6.

5. Sandroni C, Cavallaro F, Callaway CW, Sanna T, D'Arrigo S, Kuiper M, Della Marca G, Nolan JP. Predictors of poor neurological outcome in adult comatose survivors of cardiac arrest: a systematic review and meta-analysis. Part 1: patients not treated with therapeutic hypothermia. Resuscitation 2013;84:1310-23.

6. Young GB. Clinical practice. Neurologic prognosis after cardiac arrest. N Engl J Med 2009;361:60511.

7. Cloostermans MC, van Meulen FB, Eertman CJ, Hom HW, van Putten MJ. Continuous electroencephalography monitoring for early prediction of neurological outcome in postanoxic patients after cardiac arrest: a prospective cohort study. Crit Care Med 2012;40:2867-75.

8. Sandroni C, Cariou A, Cavallaro F, et al. Prognostication in comatose survivors of cardiac arrest: an advisory statement from the European Resuscitation Council and the European Society of Intensive Care Medicine. Resuscitation 2014;85:1779-89.

9. Kamps MJA, Horn J, Oddo M, et al. Prognostication of neurologic outcome in cardiac arrest patients after mild therapeutic hypothermia: a meta-analysis of the current literature. Intensive Care Med 2013;39:1671-82.

10. Geocadin RG, Callaway CW, Fink EL, et al. Standards for Studies of Neurological Prognostication in Comatose Survivors of Cardiac Arrest: A Scientific Statement From the American Heart Association. Circulation. 2019;140:e517-42.

11. Metter RB, Rittenberger JC, Guyette FX, Callaway CW. Association between a quantitative CT scan measure of brain edema and outcome after cardiac arrest. Resuscitation 2011;82:1180-5.

12. Lee YH, Oh YT, Ahn HC, et al. The prognostic value of the grey-to-white matter ratio in cardiac arrest patients treated with extracorporeal membrane oxygenation. Resuscitation 2016;99:50-5.

13. Lee BK, Kim WY, Shin J, et al. Prognostic value of gray matter to white matter ratio in hypoxic and non-hypoxic cardiac arrest with non-cardiac etiology. Am J Emerg Med 2016;34:1583-8.

14. Chae MK, Ko E, Lee JH, et al. Better prognostic value with combined optic nerve sheath diameter and grey-to-white matter ratio on initial brain computed tomography in post-cardiac arrest patients. Resuscitation 2016;104:40-5.

15. Kim YH, Lee JH, Hong CK, et al. Feasibility of optic nerve sheath diameter measured on initial brain computed tomography as an early neurologic outcome predictor after cardiac arrest. Acad Emerg Med 2014;21:1121-8.

16. Ryu JA, Chung CR, Cho YH, et al. The association of findings on brain computed tomography with neurologic outcomes following extracorporeal cardiopulmonary resuscitation. Crit Care 2017;21:15. 
17. Sekhon MS, Griesdale DE, Robba C, et al. Optic nerve sheath diameter on computed tomography is correlated with simultaneously measured intracranial pressure in patients with severe traumatic brain injury. Intensive Care Med 2014;40:1267-74.

18. Rajajee V, Fletcher JJ, Rochlen LR, Jacobs TL. Comparison of accuracy of optic nerve ultrasound for the detection of intracranial hypertension in the setting of acutely fluctuating vs stable intracranial pressure: post-hoc analysis of data from a prospective, blinded single center study. Crit Care 2012;16:R79.

19. Chelly J, Deye N, Guichard JP, et al. The optic nerve sheath diameter as a useful tool for early prediction of outcome after cardiac arrest: a prospective pilot study. Resuscitation 2016;103:7-13.

20. Lee SH, Jong Yun S. Diagnostic performance of optic nerve sheath diameter for predicting neurologic outcome in post-cardiac arrest patients: A systematic review and meta-analysis. Resuscitation 2019;138:59-67.

21. You Y, Park J, Min J, et al. Relationship between time related serum albumin concentration, optic nerve sheath diameter, cerebrospinal fluid pressure, and neurological prognosis in cardiac arrest survivors. Resuscitation 2018;131:42-7.

22. Park JS, You Y, Min JH, et al. Study on the timing of severe blood-brain barrier disruption using cerebrospinal fluid-serum albumin quotient in post cardiac arrest patients treated with targeted temperature management. Resuscitation 2018;135:118-23.

23. Selhorst JB, Chen Y. The optic nerve. Semin Neurol 2009;29:29-35.

24. Robba C, Santori G, Czosnyka M, et al. Optic nerve sheath diameter measured sonographically as non-invasive estimator of intracranial pressure: a systematic review and meta-analysis. Intensive Care Med 2018;44:1284-94.

25. Lee $\mathrm{DH}$, Lee $\mathrm{SH}, \mathrm{Oh} \mathrm{JH}$, et al. Optic nerve sheath diameter measured using early unenhanced brain computed tomography shows no correlation with neurological outcomes in patients undergoing targeted temperature management after cardiac arrest. Resuscitation 2018;128:144-50.

26. Kim DH, Jun JS, Kim R. Ultrasonographic measurement of the optic nerve sheath diameter and its association with eyeball transverse diameter in 585 healthy volunteers. Sci Rep 2017;7:15906.

27. Wang L, Feng L, Yao Y, et al. Ultrasonographic Evaluation of Optic Nerve Sheath Diameter among Healthy Chinese Adults. Ultrasound Med Biol 2016;42:683-8.

28. Maude RR, Hossain MA, Hassan MU, et al. Transorbital sonographic evaluation of normal optic nerve sheath diameter in healthy volunteers in Bangladesh. PLoS One 2013;8:e81013.

29. Romagnuolo L, Tayal V, Tomaszewski C, Saunders T, Norton HJ. Optic nerve sheath diameter does not change with patient position. Am J Emerg Med 2005;23;686-8.

30. Bhandari D, Udupi Bidkar P, Adinarayanan S, Narmadhalakshmi K, Srinivasan S. Measurement of changes in optic nerve sheath diameter using ultrasound and computed tomography scan before and after the ventriculoperitoneal shunt surgery in patients with hydrocephalus - A prospective observational trial. Br J Neurosurg 2019;33:125-30. 
31. Kang C, Min JH, Park JS, et al. Relationship between optic nerve sheath diameter measured by magnetic resonance imaging, intracranial pressure, and neurological outcome in cardiac arrest survivors who underwent targeted temperature management. Resuscitation 2019;145:43-9.

32. Geeraerts T, Newcombe VF, Coles JP, et al. Use of T2-weighted magnetic resonance imaging of the optic nerve sheath to detect raised intracranial pressure. Crit Care 2008;12:R114.

33. Kim DH, Jun JS, Kim R. Measurement of the optic nerve sheath diameter with magnetic resonance imaging and its association with eyeball diameter in healthy adults. J Clin Neurol 2018;14:345-50.

\section{Figures}

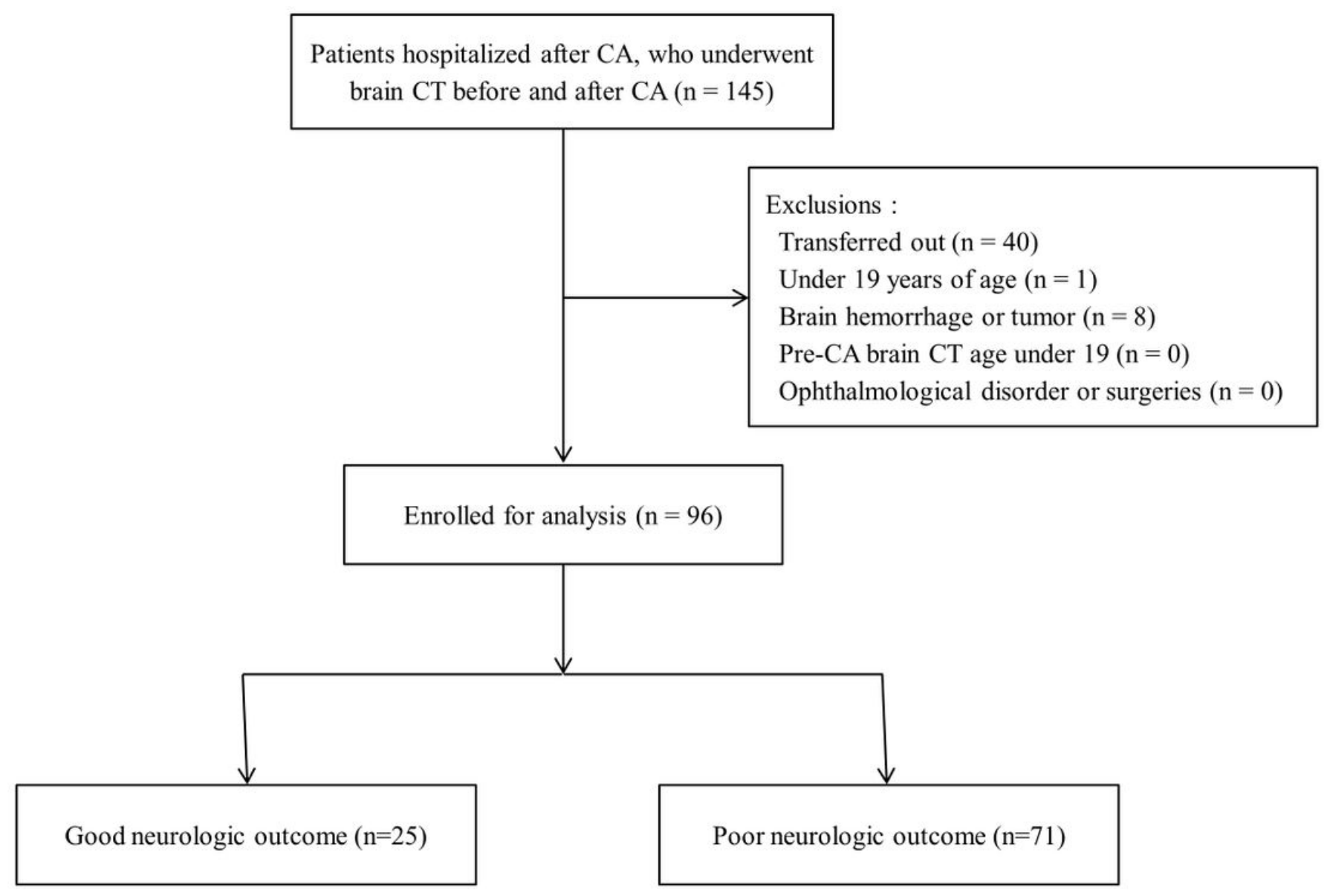

\section{Figure 1}

Flow chart of the study process 


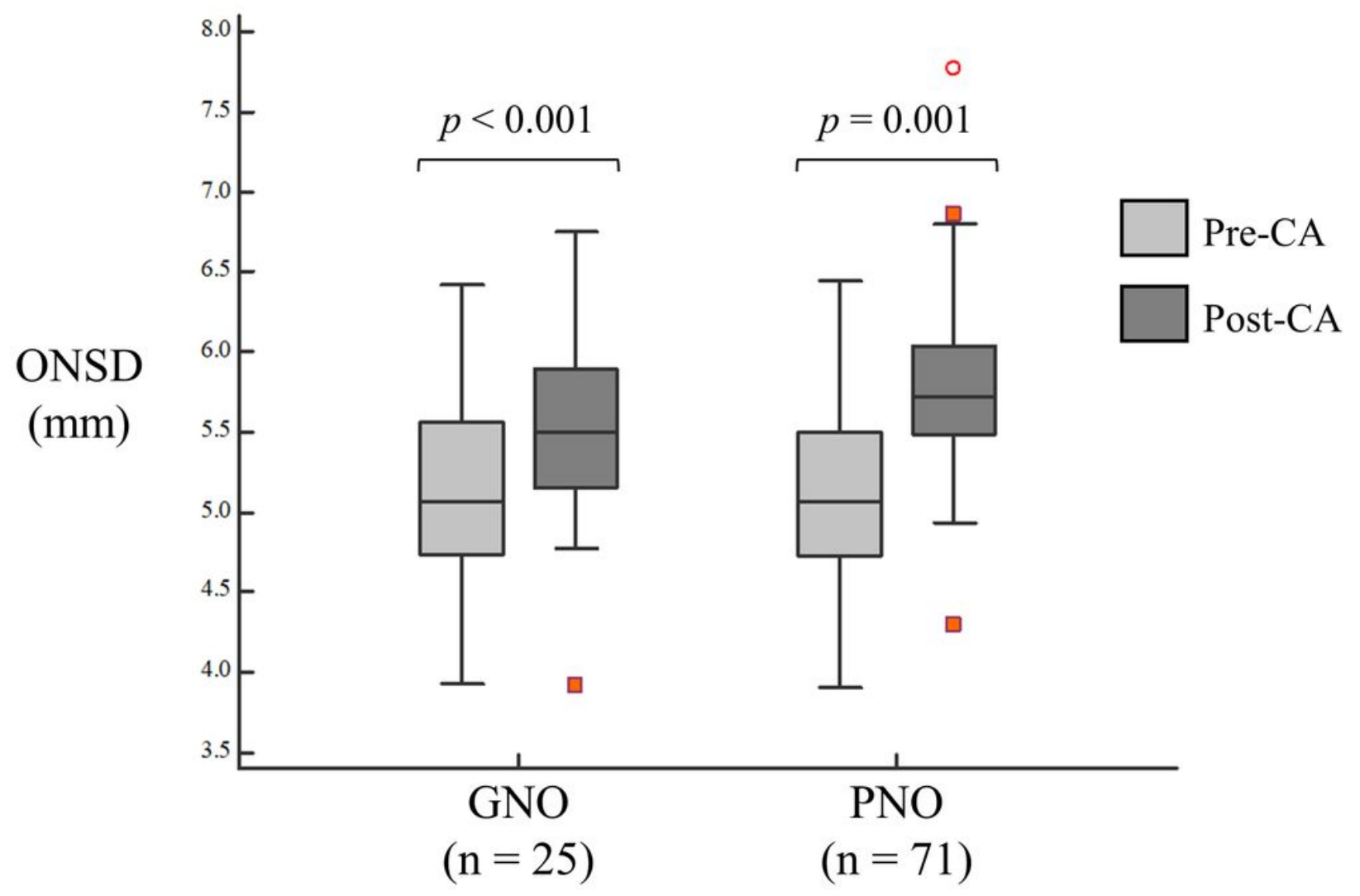

Figure 2

Comparison of the optic nerve sheath diameter between pre-cardiac arrest and post-cardiac arrest in good and poor neurologic outcome group. 


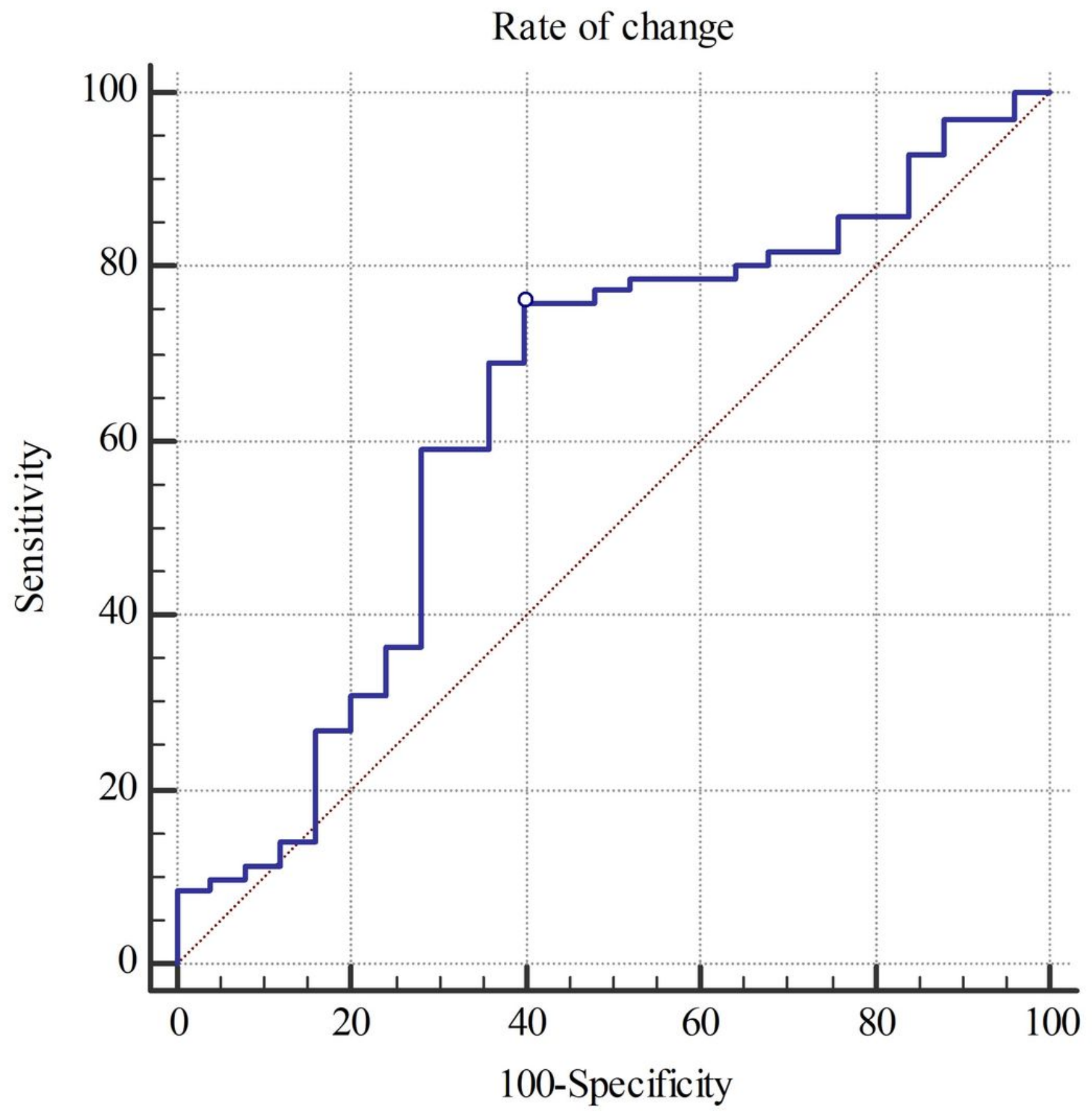

Figure 3

Receiver operator curve for predicting poor neurologic outcome using rate of optic nerve sheath diameter change. AUC $=0.64(95 \%$ Confidence interval $=0.53-0.73)$

\section{Supplementary Files}


This is a list of supplementary files associated with this preprint. Click to download.

- SuppleTable1.docx 